\title{
Transfer reactions as a tool for nuclear astrophysics
}

\section{F. Hammache*}

Institut de Physique Nucléaire d'Orsay, UMR8608, IN2P3-CNRS, Université Paris sud 11, 91406 Orsay, France

E-mail: hammachedipno.in2p3.fr

The cross section of the nuclear reactions involved in stellar nucleosynthesis are often very difficult to measure directly at stellar energies because of their very low value and/or the radiaoctive nature of the concerned isotopes, often far from the valley of stability. Moreover, this situation can be complicated by the existence of very low energy resonances and/or subthreshold resonances. Indirect methods such as transfer reactions and the ANC method offer the possibility to overcome these difficulties. In this context, the transfer reaction method will be presented and then illustrated through the study of two cases, a resonant reaction case, ${ }^{12} \mathrm{C}(\alpha, \gamma){ }^{16} \mathrm{O}$ and a direct $(\mathrm{n}, \gamma)$ capture case, ${ }^{60} \mathrm{Fe}(\mathrm{n}, \gamma){ }^{61} \mathrm{Fe}$

VI European Summer School on Experimental Nuclear Astrophysics, ENAS 6

September 18-27, 2011

Acireale Italy

${ }^{*}$ Speaker. 


\section{Introduction}

One of the main characteristics of the nuclear reactions involved in stellar nucleosynthesis is the low energy where they occur (between few $\mathrm{keV}$ to few $\mathrm{MeV}$ ) implying very small cross sections, ranging from hundreds of pico-barn to fbarn especially when it involves charged particles. These features make the direct measurements at stellar energies very difficult and often impossible. Hence, direct measurements are usually performed at higher energies and then extrapolated down to stellar energies using Rmatrix calculations. However, these extrapolations are not always free from problems. In some cases, they can even lead to wrong results because they don't take into account the contributions of a possible unseen low energy resonances such in ${ }^{22} \mathrm{Ne}(\alpha, \mathrm{n}){ }^{25} \mathrm{Mg}$ [ [3] ] reaction or a possible sub-threshold resonances like in ${ }^{13} \mathrm{C}(\alpha, \mathrm{n}){ }^{16} \mathrm{O}$ [四] and ${ }^{12} \mathrm{C}(\alpha, \gamma){ }^{16} \mathrm{O}$ [[5] reactions. The effect of these resonances may change the extrapolated $\mathrm{S}$-factor at stellar energies by a huge factor, it can be even order of magnitudes. The other problem concerning the direct measurements is related to the radioactive nature of the nuclei involved in reactions occurring in explosive sites (novae, supernovae, $\mathrm{X}$-Ray bursts, ...) and those involved in $(n, \gamma)$ radiative captures in r-process and sometimes in s-process like ${ }^{60} \mathrm{Fe}(\mathrm{n}, \gamma)$ reactions. The intensities of the radioactive beams are often low, rarely exceeding $10^{5}$ to $10^{6} \mathrm{pps}$ and for nuclei with relatively long half life, making targets with enough atoms per $\mathrm{cm}^{2}$ is very difficult. Hence the direct measurements of such reactions is very difficult and challenging and in case of r-process reactions it is currently impossible. To bypass these difficulties (sub-threshold resonances, radioactive nuclei,...) indirect methods such as transfer reactions are good alternatives. In these methods, the experiments are usually performed at high energies implying higher cross sections and the conditions are relatively less rigorous than in direct measurements (target thickness and composition, high background, ...) . However, these methods are model dependent. They depend, as we will see in section 2 , on the uncertainties relative to the different parameters used in the model. Hence, there are two sources of errors, experimental and theoretical. But the global uncertainty on the measured cross section directly or indirectly can be reduced by combining different approaches and methods. There are different indirect methods that were used to overcome the problems mentioned above: the transfer reactions, the coulomb dissociation method, the ANC method, the elastic and inelastic scattering and finally the Trojan Horse method. Here, I will focus on the transfer reaction method and I will show the possible type of reactions one can study using this method: the resonant one and the non resonant direct radiative capture reaction.

\subsection{Resonant reactions}

A resonant reaction, as shown in Fig.1, is a two step process where the entrance channel $A+x$ forms first an excited state $\mathrm{E}_{x}$ in the compound nucleus $\mathrm{C}$ at the incident energy $\mathrm{E}_{c m}$ and then the formed resonant state decays either to the exit channel $(\mathrm{B}+\mathrm{y})$ or to its ground state by emitting $\gamma$-rays. The resonant process can occur only at energies where the incident energy in $\mathrm{cm}$ is very close to the resonance energy which is given by: $\mathrm{E}_{r}=\mathrm{E}_{x}-\mathrm{Q}$ where $\mathrm{Q}$ is the $\mathrm{Q}$-value of the reaction.

Hence, in case of a narrow resonance $\left(\Gamma_{t o t} \ll \mathrm{E}_{r}\right)$, the reaction rate is given by:

$$
<\sigma v>=\left(\frac{2 \pi}{\mu k T}\right)^{3 / 2}(\omega \gamma)_{R} \exp \left(-\frac{E_{R}}{k T}\right)
$$




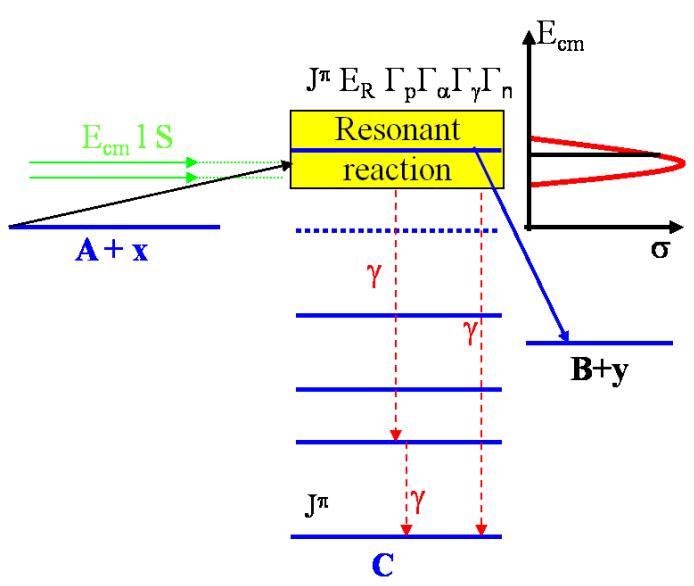

Figure 1: Resonant reaction scheme

where the resonance strength is given by:

$$
(\omega \gamma)_{R}=\frac{2 J_{C}+1}{\left(2 J_{A}+1\right)\left(2 J_{x}+1\right)} \frac{\Gamma_{x} \Gamma_{y}}{\Gamma_{t o t}}
$$

The important parameters to describe the resonant mechanism and calculate the reaction rate are the resonance energy $\mathrm{E}_{r}$, the partial width $\Gamma_{x}$ which expresses the probability to form the compound nucleus $\mathrm{C}^{*}$ in the excited state $\mathrm{E}_{x}$, the partial width $\Gamma_{y}$ which expresses the probability of decay to the exit channel $\mathrm{B}+\mathrm{y}$ or $\mathrm{C}+\gamma$ and the spin-parity of the nuclei and states involved.

Once these different parameters are known, one can easily calculate the reaction rate using formulae (1.1). The exciatation energy and the partial decay widths of the states of interest can be obtained from transfer reactions experiments.

Let's assume a compound nucleus $\mathrm{C}$ in an excited state $\mathrm{E}_{r}$ (see fig2) a pure core-particle configuration $|\mathrm{A} \otimes \mathrm{x}\rangle$.

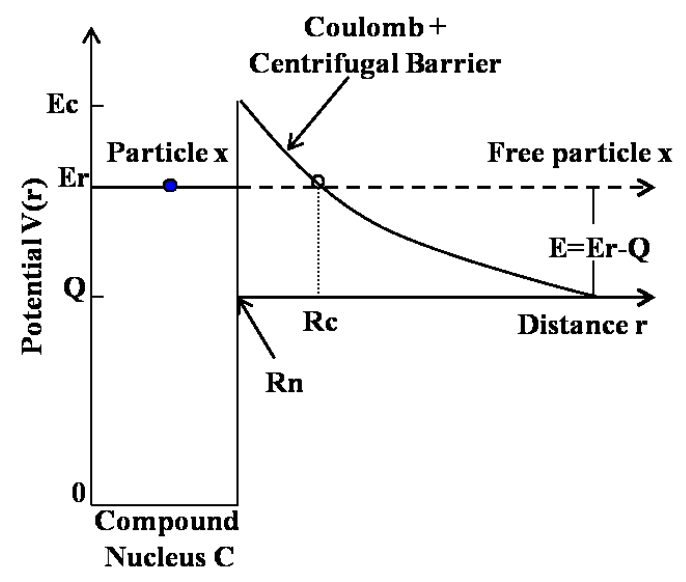

Figure 2: 
Thanks to the time reverse invariance of the electro-magnetic and nuclear processes, the partial width of the formation of the compound nucleus in the excited state $\mathrm{E}_{r}$ through $\mathrm{A}+\mathrm{x}$ is identical to the partial width of the decay of the excited state $\mathrm{C}^{*}$ into $\mathrm{A}+\mathrm{x}$. The decay partial width of $\mathrm{C}$ into $\mathrm{A}+\mathrm{x}$ is given by the following formulae:

$$
\Gamma_{x}^{s . p}=\frac{\hbar^{2}}{\mu} R P_{l}(E, R)|\varphi(R)|^{2}
$$

with

$$
\left.\int_{0}^{\infty} \varphi(R)\right|^{2} R^{2} d R=1
$$

Where $\mathrm{R}$ is is the channel radius, $\mathrm{P}_{l}$ is the penetrability factor, $\mathrm{E}$ is the energy of the free particle $\mathrm{x}$ and $\varphi(\mathrm{R})$ is the radial wave function of the particle $\mathrm{x}$. So for a state with a pure core particle configuration, the single particle $\Gamma_{x}^{s . p}$ can be calculated. But in most cases, they are not pure coreparticle configurations, they are a mixture of configurations which decay or formation partial width $\Gamma_{x}$ is given by the product of the single particle $\Gamma_{x}^{s p}$ and the spectroscopic factor $\mathrm{S}: \Gamma_{x}=\Gamma_{x}^{s p} \times \mathrm{C}^{2} \mathrm{~S}$ where $\mathrm{C}$ is the isospin Clebsch-Grodon coefficient. The spectroscopic factor $\mathrm{S}$ expresses the overlap probability between the antisymmetrized wave functions of the entrance channel $A+x$ and the final state $\mathrm{B} ; \mathrm{S}=<\mathrm{A}+\mathrm{x} \mid \mathrm{B}>$. Hence, by determining the spectroscopic factor $\mathrm{S}$ via transfer reactions, one can then calculate $\Gamma_{x}$ or the reduced width as we will see in section 2.

\subsection{Non-resonant reactions: the $(n, \gamma)$ case}

For reactions where direct capture mechanism can sometime plays an important role such as in ${ }^{48} \mathrm{Ca}(\mathrm{n}, \gamma){ }^{49} \mathrm{Ca}$ reaction [焑], the capture occurs on bound states of the final nucleus in a one step process (see fig 3). The captures are possible at all neutron energies and the cross section varies smoothly with the neutron energy.

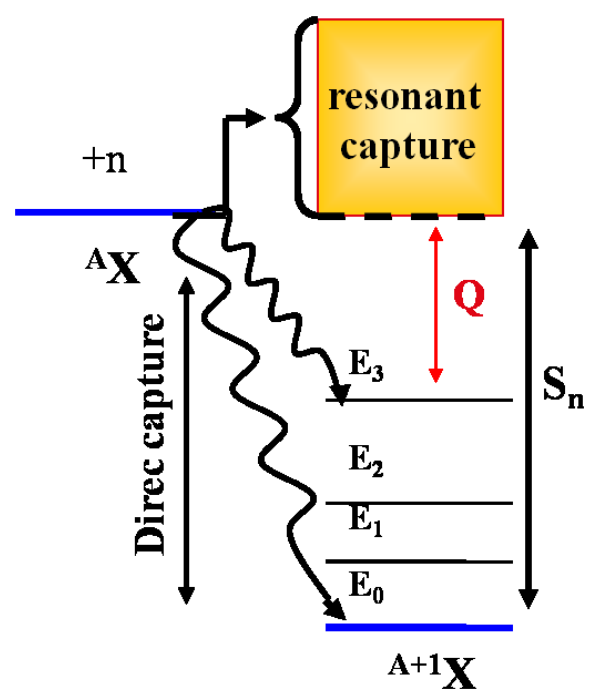

Figure 3: Direct capture reaction scheme 
The cross section of the direct process is described by the following single matrix element [ [6]:

$$
\sigma_{(n, \gamma)}=\sum_{i} C_{i}^{2} S_{i}\left|\int_{r=0}^{\infty} \phi_{f} \theta_{e m} \phi_{i} d \vec{r}\right|^{2}
$$

Where $\theta_{e m}$ is the electro-magnetic multipole transition operator, $\phi_{i}$ and $\phi_{f}$ are the scattering and the bound state wave functions in the entrance and exit channels, respectively. The wave functions are Schrödinger's equation solutions with the potential V obtained by double folding and $\mathrm{S}$ is the spectroscopic factor of the final state. The double folding potential $\mathrm{V}$ is given by:

$$
V=\lambda \iint \rho_{n}\left(\vec{r}_{n}\right) \rho_{A}\left(\vec{r}_{A}\right) v_{e f f}\left(E_{n}, \rho_{n}, \rho_{A},|\vec{R}-\vec{r}|\right) d \vec{r}_{n} d \vec{r}_{A}
$$

where $\mathrm{v}_{\text {eff }}$ is the nucleon-nucleon interaction, $\rho_{n}$ is the neutron density, $\rho_{A}$ is the nucleus target density and $\lambda$ is a factor adjusted to reproduce the elastic scattering data for the entrance channel and the neutron separation energy for the exit channel. The latter depends on the location of the excited states in the final nucleus. These spectroscopic information on the low energy bound states $\left(\mathrm{E}_{x}, 1, \mathrm{C}^{2} \mathrm{~S}\right)$ are accessible via $(\mathrm{d}, \mathrm{p})$ transfer reactions in case of $(\mathrm{n}, \gamma)$ reaction, for example.

\section{Transfer Reaction Method}

Let's assume that we want to study a resonant reaction $\mathrm{x}+\mathrm{A} \rightarrow \mathrm{C}^{*} \rightarrow \mathrm{B}+\mathrm{y}$ and measure the partial decay width $\Gamma_{x}$ of the state of interest in $\mathrm{C}^{*}$ into the entrance channel $\mathrm{A}+\mathrm{x}$. To do so, we populate the excited states of $\mathrm{C}$ by transfering the small particle $\mathrm{x}$ (see figure 4) which can be a nucleon or a cluster of nucleons from the nucleus $\mathrm{X}$ to the nucleus $\mathrm{A}$.

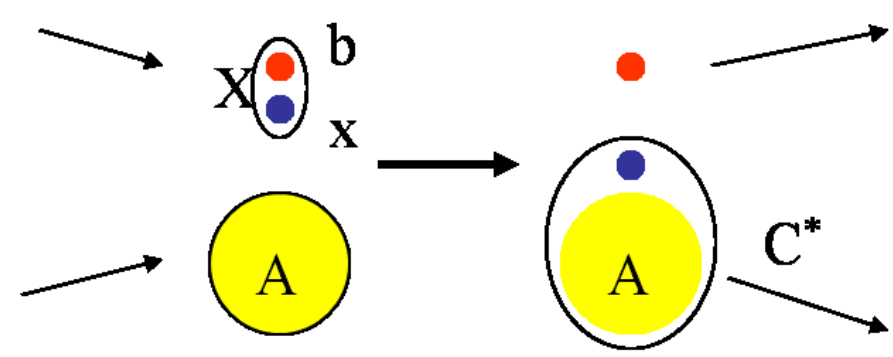

Figure 4: Sketch of a transfer reaction before (left) and after reaction (right).

This will feed the valence states of the final nucleus $\mathrm{C}$, hopefully with no perturbation of the core, that is why it is called one step direct transfer reaction. The other part of the projectile $b$ will continue its movement and will be detected. By measuring the emitted angle and energy of the particle $\mathrm{b}$, one can deduce the excited state that was populated in $\mathrm{C}$ from kinematics and by comparing the shape of the measured angular distributions to those predicted by the distorted Born approximation theory (DWBA), one can deduce the angular orbital momentum 1 of the populated state. The theoretical direct transfer cross section is calculated using the DWBA formalism and it is given by the following matrix element:

$$
\left(\frac{d \sigma}{d \Omega}\right)_{D W B A} \propto\left|<\chi_{f} I_{x A}^{C}\left(r_{x A}\right)\right| V\left|I_{b x}^{X}\left(r_{b x}\right) \chi_{i}>\right|^{2}
$$


Where $\chi_{i, f}$ are the distorted wave functions of the initial and the final state, $\mathrm{V}$ is the transition transfer operator, $\mathrm{I}_{x A}^{C}\left(\mathrm{r}_{x A}\right)$ is the overlap function of the final bound state $\mathrm{C}$ formed by $\mathrm{A}+\mathrm{x}$ and $\mathrm{I}^{X}{ }_{b x}\left(\mathrm{r}_{b x}\right)$ is the overlap function of the bound state $\mathrm{X}$ formed by $\mathrm{b}+\mathrm{x}$.

The radial part of these last functions is given by the following product:

$$
I_{\beta \gamma}^{\alpha}\left(r_{\beta \gamma}\right)=S^{1 / 2} \varphi_{\beta \gamma}\left(r_{\beta \gamma}\right)
$$

Where $\mathrm{S}$ is the spectroscopic factor, $\varphi_{\beta \gamma}(\mathrm{r})$ is the radial wave function of the bound state $\mathrm{C}$ or $\mathrm{X}$ with $\alpha$ being the final bound state $\mathrm{C}$ or the bound state $\mathrm{X}, \beta$ being the transferred particle $\mathrm{x}$ and $\gamma$ being A or b respectively.

The spectroscopic factor of the different populated states in $\mathrm{C}$ can be extracted from the ratio of the measured differential cross section to the one calculated by DWBA:

$$
\left(\frac{d \sigma}{d \Omega}\right)_{\exp }=S_{1} S_{2}\left(\frac{d \sigma}{d \Omega}\right)_{D W}
$$

As one can see in formulae 2.3, there are two spectroscopic factors, $S_{1}$ for the final bound state of interest in the exit channel and $\mathrm{S}_{2}$ for the bound state in the entrance channel. Hence, knowing one of the spectroscopic factors it is possible to extract the other one. Once the spectroscopic factor of the state of interest is extracted, one can then determine the reduced decay width using the following formula [D]:

$$
\gamma_{x}^{2}=\frac{\hbar^{2} R}{2 \mu} S_{x}|\varphi(R)|^{2}
$$

where $\varphi(\mathrm{R})$ is the radial wave function of the bound state $\mathrm{C}$ formed by $\mathrm{A}+\mathrm{x}$, calculated at a channel radius $\mathrm{R}$ where $\varphi(\mathrm{R})$ has its asymptotic behavior. Then the calculation of $\Gamma_{x}$ is possible using:

$$
\Gamma_{x}=2 P_{l} \gamma_{x}^{2}
$$

One can also extract the ANC [8] using the expression:

$$
\tilde{C}^{2}=S_{\alpha} \frac{R^{2} \varphi^{2}(R)}{\tilde{W}^{2}(R)}
$$

where $\mathrm{W}$ is the Whittaker function.

To calculate the transfer DWBA differential cross sections, some inputs are needed and they have to be carefully determined. These inputs are the optical potential parameters describing the entrance channel, those describing the exit channel and finally the potential well parameters describing the interaction of the transferred particle with the core in the final nucleus. For the optical potential parameters of the entrance and exit channel, elastic scattering measurements are needed. When elastic measurements are not available, one can use the potentials from measurements performed in the mass region close to the nuclei of interest and at close incident energies or the global potential formulae from Perey and Perey [Q], Daehnick et al. [ए]],...etc. Concerning the well parameters, different realistic sets of potentials can be used and the selected ones are those giving the optimal fit of the measured angular distributions. Note that the accuracy on the extraction of the 
spectroscopic factor can hardly be better than $30 \%$, so this method can not be used for reactions where an uncertainty better than $10 \%$ is needed.

The calculation of the transfer reaction cross sections with DWBA formalism involves the evaluation of a six dimensional integral [W]. For light-ion reactions such as (d,p) reaction, the direct reaction causing the transition is often approximated by a zero-range potential. This is partially justified by the small size of the light nucleus (the deuteron in the example cited above) in comparison with the size of the other nuclei and the $S$ wave state of the projectile. With this assumption, the DWBA integrals reduce to three-dimensional integrals that make the calculation simpler as only the form factor describing the interaction of the transferred particle with the core in the final nucleus has to be considered. However, this zero range assumption is no more valid if the projectile is not in an $\mathrm{S}$ wave internal state $(\mathrm{L}=0)$ and in case of reactions where the transferred particle is a cluster of more than 2 nucleons such as the $\alpha$-particle. Here, the relatively large size of the nucleus $\left({ }^{6} \mathrm{Li}\right.$ or ${ }^{7} \mathrm{Li}$ in case of $\alpha$ transfer) from which the cluster is transferred, together with the small wavelength associated with the relative motion, makes this assumption very poor and sometimes wrong. For these cases, finite range DWBA calculations have to be performed with a six dimensional integral which includes both form factors describing the interaction of the transferred particle with the core in the initial nucleus and in the final nucleus respectively.

When using the transfer method, one has to be aware that other reaction mechanisms such as the compound nucleus mechanism or the multi-step transfer reaction mechanism [ए]] can occur. The contribution of these mechanisms can be evaluated by using Hauser Feschbach calculations and coupled channel calculations respectively [102]. Note that usually the direct transfer mechanism is favored at small centre-of-mass angles $\left(\theta_{c . m} \leqslant 50^{\circ}\right)$, so one has to perform the transfer experiments at detection angles and incident energies where the compound nucleus component is reduced.

An illustration of the method will be given in the following through the study of two reaction cases, the resonant reaction ${ }^{12} \mathrm{C}(\alpha, \gamma){ }^{16} \mathrm{O}$ which was studied via ${ }^{12} \mathrm{C}\left({ }^{7} \mathrm{Li}, \mathrm{t}\right)^{16} \mathrm{O}$ and the direct capture reaction ${ }^{60} \mathrm{Fe}(\mathrm{n}, \gamma){ }^{61} \mathrm{Fe}$ which was studied via d $\left({ }^{60} \mathrm{Fe}, \mathrm{p}\right){ }^{61} \mathrm{Fe}$.

\section{Application of the method to a resonant case: ${ }^{12} \mathbf{C}(\alpha, \gamma){ }^{16} \mathrm{O}$}

${ }^{12} \mathrm{C}(\alpha, \gamma){ }^{16} \mathrm{O}$ reaction plays an important role in helium burning in massive stars and their evolution [U, []]. However, the low-energy cross section of ${ }^{12} \mathrm{C}(\alpha, \gamma){ }^{16} \mathrm{O}$ remains highly uncertain despite the various experiments performed during the last four decades. ${ }^{12} \mathrm{C}(\alpha, \gamma){ }^{16} \mathrm{O}$ reaction occurs at temperature of about $0.2 \mathrm{GK}$ that corresponds to a Gamow peak of about $300 \mathrm{keV}$. At this energy, the expected cross section is extremely low, about $10^{-8}$ nbarn, impossible to measure directly. Direct measurements were performed down to $900 \mathrm{keV}$ in the center-of-mass system which is far from the energy of interest. The extrapolation of the measured cross sections to stellar energies $(E=300 \mathrm{keV})$ is made difficult by the presence of the two sub-threshold states at 6.92 $\left(2^{+}\right)$and $7.12\left(1^{-}\right) \mathrm{MeV}$ of ${ }^{16} \mathrm{O}$. The high energy tails of these two sub-threshold resonances can increase the cross section but their effect is badly known because their reduced alpha width and so their corresponding alpha spectroscopic factors $S_{\alpha}$ are spread over a large range of values [可].

A recent measurement of the $S_{\alpha}$ of the two subthreshold states at $7.12 \mathrm{MeV}$ and $6.92 \mathrm{MeV}$ of ${ }^{16} \mathrm{O}$ was performed through the transfer reaction ${ }^{12} \mathrm{C}\left({ }^{7} \mathrm{Li}, \mathrm{t}\right){ }^{16} \mathrm{O}$ at two incident energies, 28 and 34 $\mathrm{MeV}$ respectively, at the Tandem-Alto facility of Orsay. The tritons were analyzed with an Enge 
Split-pole magnetic spectrometer and detected at the focal plane by a $50 \mathrm{~cm}$ long position-sensitive gas chamber and a $\Delta \mathrm{E}$ proportional gas-counter. The tritons were detected at angles ranging from 0 to 31 degrees corresponding to angles up to 43 degrees in the center of mass frame.

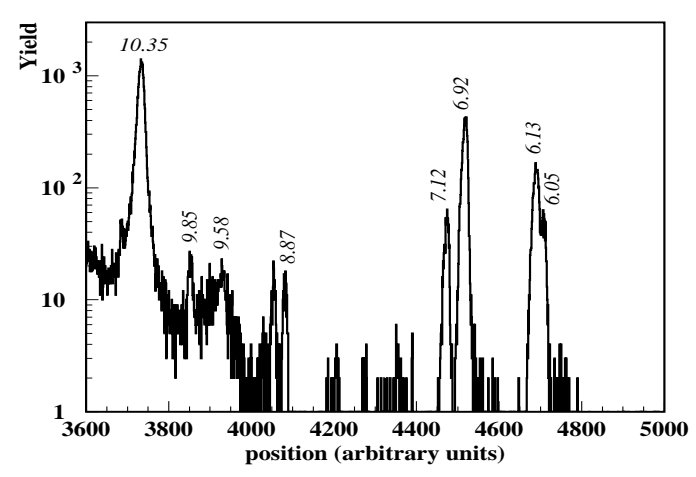

Figure 5: Triton spectrum, in the excitation energy region from 6 to $11 \mathrm{MeV}$, obtained at $11.5^{\circ}$ (lab) with the $34 \mathrm{MeV}^{7} \mathrm{Li}$ beam on ${ }^{12} \mathrm{C}$ target. The excitation energy $(\mathrm{MeV})$ of ${ }^{16} \mathrm{O}$ levels are indicated

Angular distribution measurements were performed at two incident energies, 28 and $34 \mathrm{MeV}$, in order to check the direct character of the transfer mechanism. Elastic scattering measurements were also performed at $28 \mathrm{MeV}$ incident energy in order to get realistic optical potential parameters for the entrance channel. Typical excitation energy spectrum of ${ }^{16} \mathrm{O}$ measured at $11^{\circ}$ is displayed in figure 5. The overall energy resolution obtained was about $50 \mathrm{keV}$.

The experimental differential cross section measured for the 6.05, 6.13, 6.92 and $7.12 \mathrm{popu}-$ lated states of ${ }^{16} \mathrm{O}$ at the two incident energies of $34 \mathrm{MeV}$ and $28 \mathrm{MeV}$ are displayed in figures 6 left and right, respectively, together with the finite range DWBA calculations and the Hauser Feshbach (HF) calculations, which were performed to evalutate the compound nuclear reaction contribution. The HF calculations were performed by considering the triton from $\left({ }^{7} \mathrm{Li}, \mathrm{t}\right)$ as coming from evaporation of the compound nucleus ${ }^{19} \mathrm{~F}$ and they were normalized by a factor extracted from the ratio of the absolute values of the compound nucleus cross sections calculated for the observed non-natural parity $1^{-}(8.87 \mathrm{MeV})$ state to those measured in this experiment [15]].

The finite-range DWBA calculations were performed using Fresco code [ए13]. Many combinations of entrance and exit optical potentials parameters were investigated. Concerning the entrance channel, several ${ }^{7} \mathrm{Li}+{ }^{12} \mathrm{C}$ optical potentials extracted from elastic measurements were tested. For the exit triton channel, optical potential parameters from Garrett et al [प14] were selected. The dependence of the calculation to the ${ }^{12} \mathrm{C}-\alpha$ interaction potential was also investigated. A spreading of about $30 \%$ on $\mathrm{S}_{\alpha}$ was found when the well geometry parameters were varied. Finally, the selected optical and interaction parameters are those giving the best fit, using the maximum likelihood function set at $3 \sigma$ level, for the studied states $(6.05,6.13,6.92,7.12 \mathrm{MeV})$ at both incident energies. A good agreement between the DWBA calculations and the measured differential cross sections of the different excited states of ${ }^{16} \mathrm{O}$ can be observed at the two bombarding energies which gives strong evidence of the direct nature of $\left({ }^{7} \mathrm{Li}, \mathrm{t}\right)$ transfer reaction.

From a $\chi^{2}$ minimization of the DWBA differential cross sections to the measured ones, $\mathrm{S}_{\alpha}$ mean values of $0.15 \pm 0.05$ and $0.07 \pm 0.03$ are deduced for the states of interest at $6.92 \mathrm{MeV}$ and 

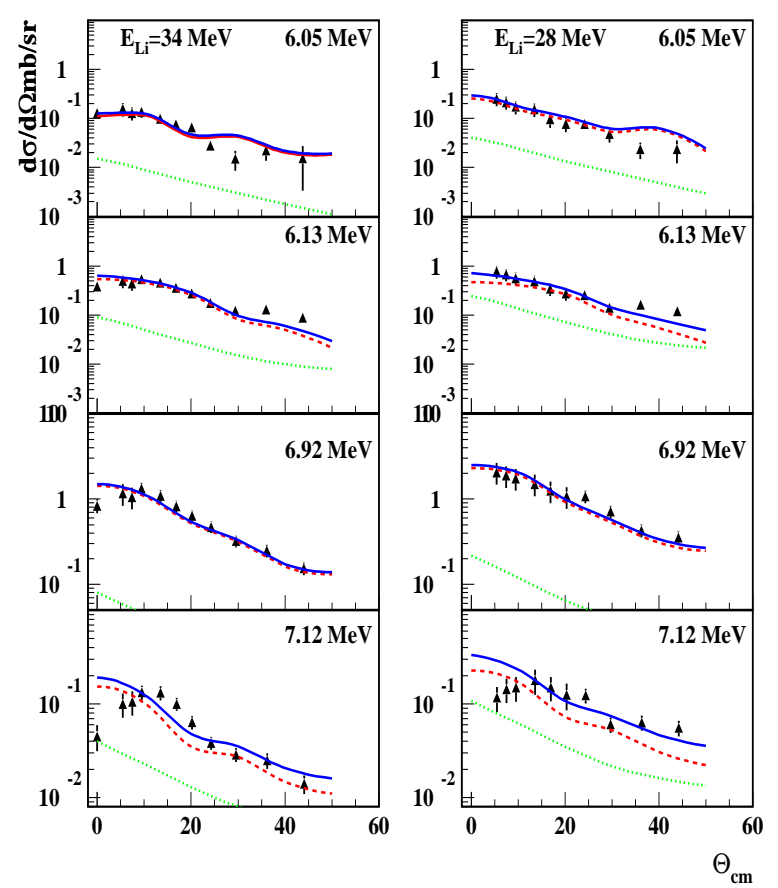

Figure 6: Experimental differential cross sections of the ${ }^{12} \mathrm{C}\left({ }^{7} \mathrm{Li}, \mathrm{t}\right){ }^{16} \mathrm{O}$ reaction obtained at 34 and $28 \mathrm{MeV}$ for the 6.05, 6.13, 6.92 and 7.12 MeV states, compared with FRDWBA calculations (red curve) normalized to the data, Hauser-Feshbach (HF) calculations (green) and the sum HF+FRDWBA (Blue).

7.12 $\mathrm{MeV}$ of ${ }^{16} \mathrm{O}$ respectively. The $\alpha$-reduced width $\gamma_{\alpha}^{2}$ of $26.70 \pm 10.30 \mathrm{keV}$ and $7.8 \pm 2.7 \mathrm{keV}$ for the $6.92 \mathrm{MeV}$ and 7.12 states respectively were then obtained at the radius of $6.5 \mathrm{fm}$ using equation 2.4. They were then included in an R-matrix calculation of the E1 and E2 component of ${ }^{12} \mathrm{C}(\alpha, \gamma){ }^{16} \mathrm{O}$ using Pierre Descouvemont R-matrix code.

From the E2 and E1 R-matrix fits [ए6, ए5] of both ${ }^{12} \mathrm{C}(\alpha, \gamma){ }^{16} \mathrm{O}$ astrophysical S-factors obtained by direct measurements at high energies and the phase shifts data from elastic scattering ${ }^{12} \mathrm{C}(\alpha, \alpha){ }^{12} \mathrm{C}$ measurements, an E2-Sfactor of $50 \pm 19 \mathrm{keV}-\mathrm{b}$ and an E1-Sfactor of $100 \pm 28 \mathrm{keV}-\mathrm{b}$ were obtained at the energy of interest $\mathrm{E}_{c m} \sim 300 \mathrm{keV}$. If we take for the cascade S-factor the value $25_{-15}^{+16} \mathrm{keV}-\mathrm{b}$ from [ㄱ] , we obtain a total S-factor, $\mathrm{S}(300 \mathrm{keV})=175 \pm 63 \mathrm{keV}-\mathrm{b}$.

\section{Application of the method to a non-resonant case: ${ }^{60} \mathrm{Fe}(\mathrm{n}, \gamma){ }^{61} \mathrm{Fe}$}

The observation of ${ }^{60} \mathrm{Fe}$ characteristic gamma-ray lines by RHESSI and INTEGRAL space-

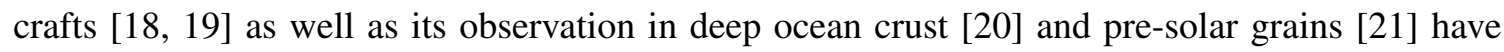
underlined the need for accurate nuclear information concerning the stellar nucleosynthesis of this nuclei. The yield of ${ }^{60} \mathrm{Fe}$, which is believed to be primarily produced in core-collapse supernovae [22, [23], is very uncertain due to the large uncertainties surrounding the cross sections of the production and destruction reactions ${ }^{59} \mathrm{Fe}(\mathrm{n}, \gamma)^{60} \mathrm{Fe}$ and ${ }^{60} \mathrm{Fe}(\mathrm{n}, \gamma){ }^{61} \mathrm{Fe}$ respectively. To evaluate, the cross sections of these two reactions, Hauser Feschbach calculations were used to estimate the resonant capture component while the direct component is determined using the equations described in 
section 1.2 and the spectroscopic informations $\left(\mathrm{E}_{x}, 1, \mathrm{C}^{2} \mathrm{~S}\right)$ of ${ }^{60} \mathrm{Fe}$ and ${ }^{61} \mathrm{Fe}$ coming from measurements and shell model calculations, respectively. Our group decided to improve, via the transfer reaction $\mathrm{d}\left({ }^{60} \mathrm{Fe}, \mathrm{p} \gamma\right){ }^{61} \mathrm{Fe}$, the spectroscopy of the poorly known ${ }^{61} \mathrm{Fe}$ needed to evaluate the direct component of ${ }^{60} \mathrm{Fe}(\mathrm{n}, \gamma){ }^{61} \mathrm{Fe}$ cross section. Indeed, this component is likely not to be negligible in comparison to the resonant one because of the relatively small $\mathrm{S}_{n}$ value $\left(\mathrm{S}_{n}=5.58 \mathrm{MeV}\right)$, which may lead to a low enough level density in contrary to a large $S_{n}$ value that, in turn, leads to a very high level density and hence to a dominant resonant component.

The (d,p $\gamma$ ) experiment was performed at LISE/GANIL using MUST2/EXOGAM experimental setup (see fig.7) [24] and an ${ }^{60} \mathrm{Fe}$ secondary beam of $10^{5}$ pps produced by fragmentation of a ${ }^{64} \mathrm{Ni}^{28+}$ primary beam at $64.5 \mathrm{~A} \mathrm{MeV}$ on a ${ }^{9} \mathrm{Be}$ target and slowed down to the energy of $27 \mathrm{~A} \mathrm{MeV}$ using a ${ }^{9} \mathrm{Be}$ degrader.

The obtained ${ }^{61} \mathrm{Fe}$ excitation energy spectrum, using the MUST2 Silicon telescopes, is displayed in figure 8 . Two peaks are obseved below the neutron threshold. The green spectrum is the contribution coming from the carbon background that was evaluated using a ${ }^{12} \mathrm{C}$ target and the blue curve is the contribution from the deuteron breakup, evaluated from a phase space monte-carlo calculation with an arbitrary normalization. The huge bump above the neutron threshold is due to the deuteron breakup events and to the population of the resonant states of ${ }^{61} \mathrm{Fe}$ above the neutron threshold. However, the detailed study of this upper part of the excitation energy spectrum needs first a good evaluation and calculation of the deuteron breakup contribution not yet done.

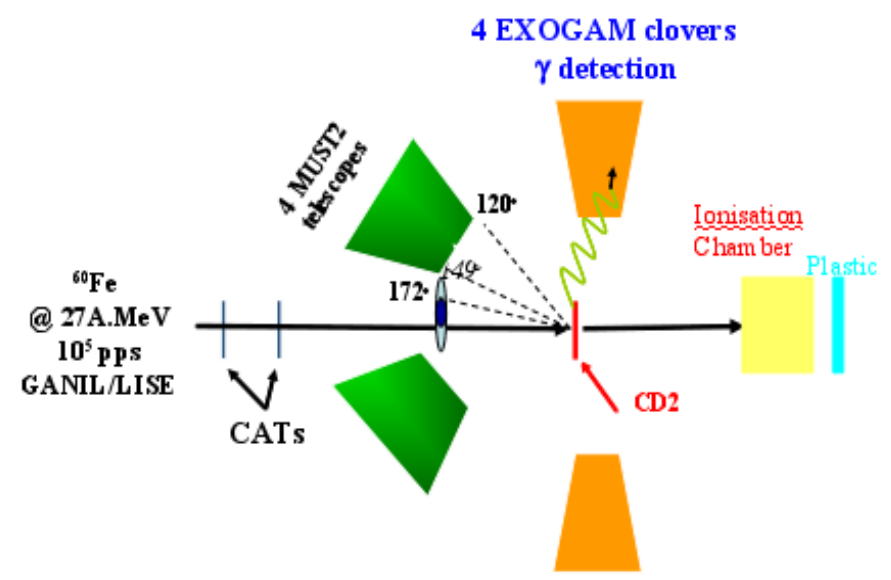

Figure 7: Experimental setup used for $\mathrm{d}\left({ }^{60} \mathrm{Fe}, \mathrm{p} \gamma\right){ }^{61} \mathrm{Fe}$ reaction.

The width of the two peaks observed below the neutron threshold was found to be $1.5 \mathrm{MeV}$ which is much larger than the expected energy resolution of about $800 \mathrm{keV}$. This means that more than one state is present in these peaks. Therefore, the $\gamma$-ray measurements helped to discriminate the different populated states in the first peak.

From the comparison of the excitation energy spectrum with and without $\gamma$-ray coincidence and from the observation of the gamma-ray spectra corresponding to different energy ranges in the first peak, three states were identified: the known $207 \mathrm{keV}$ and the isomeric state at $861 \mathrm{keV}$ and a new state, which was not observed previously, at $1401 \mathrm{keV}$. 


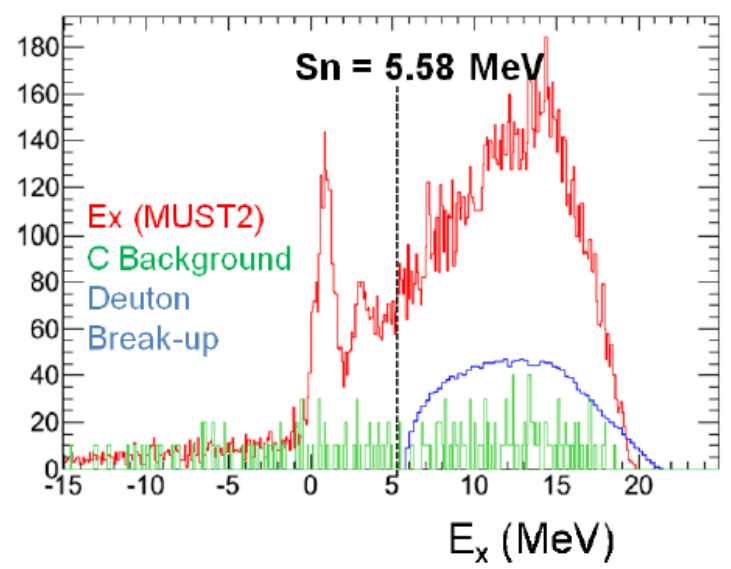

Figure 8: ${ }^{61} \mathrm{Fe}$ measured excitation energy spectrum.

A deconvolution of the three states identified in the first peak was performed in order to evaluate the yield of each populated state and extract the differential cross sections. The very preliminary measured differential cross sections for the three states at 201,861 and $1401 \mathrm{keV}$ of ${ }^{61} \mathrm{Fe}$ are displayed in figure 9 together with the zero-range DWBA calculations. For these calculations, the adiabatic approximation which takes into account the deuteron breakup [25] was used to describe the entrance channel.

The angular momentums of the 207 and $861 \mathrm{keV}$ states are already known, $\mathrm{l}=3$ (f5/2) and $\mathrm{l}=4$ (g9/2) respectively. From the $\chi^{2}$ minimization of the calculations to the data, spectroscopic factors of $0.50 \pm 0.04$ and $0.38 \pm 0.11$ were deduced for the f5/2 and $\mathrm{g} 9 / 2$ states, respectively.

Concerning the new state at $1401 \mathrm{keV}$ of ${ }^{61} \mathrm{Fe}$, its angular momentum was not known. Various calculations with different orbital momenta and different combinations were performed. Those giving the best $\chi^{2}$ fit (figure 10) are the $\mathrm{p} 3 / 2$ in blue and the $55 / 2$ in red. The spectroscopic factors obtained are respectively, $0.71 \pm 0.21$ (p3/2) and $0.32 \pm 0.09$ (f5/2), respectively.

The analysis of the experiment is still in progress. Once this is over and all results confirmed and compared to shell model calculations, calculations of the direct capture cross section using equation 1.3 with the TEDCA code [ [26] will be performed as well as the direct capture reaction rate.

\section{Conclusions}

In both resonant and non resonant capture, the spectroscopic properties $\left(\mathrm{J}^{\pi}, \mathrm{E}_{r}, \mathrm{C}^{2} \mathrm{~S}(\Gamma)\right)$ of the involved states are needed to compute the reaction rate. We have seen that transfer reaction is a good alternative method that can be used to extract spectrosopic factors, partial widths, orbital momenta and resonance energies involved in resonant and non-resonant reaction rates. The sensitivity studies of the spectroscopic factors to the potential and well parameters is necessary to evaluate the error bars and DWBA analysis of the data is improved when elastic scattering measurements in the entrance and exit channel can be performed. In this method, one should also check the dominant character of the one step direct transfer which is described by the DWBA formalism in comparison 


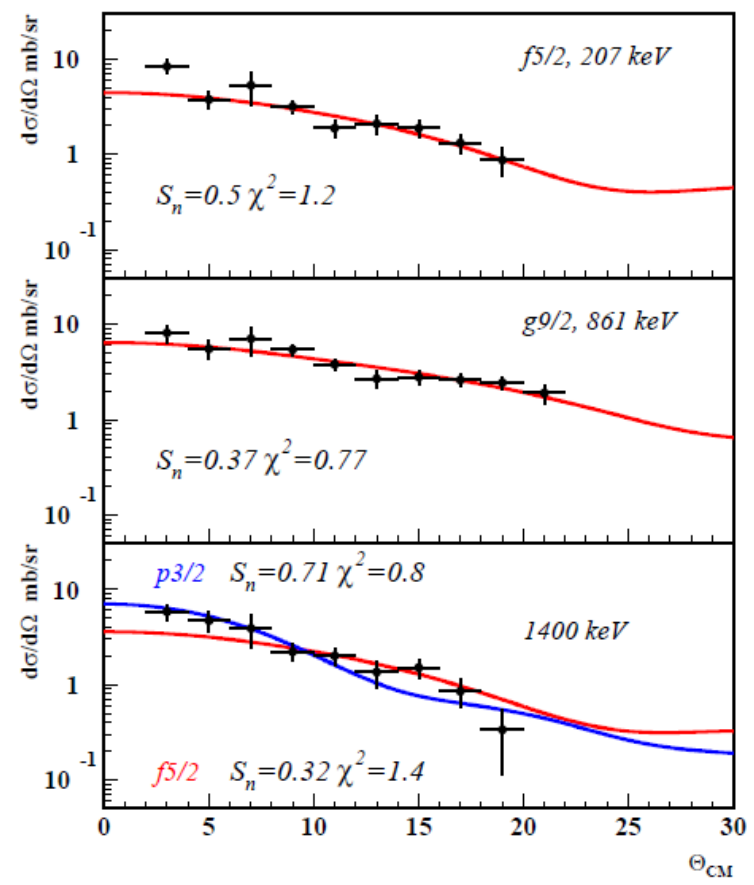

Figure 9: Preliminary experimental and theoretical differential cross sections for the 207, 861 and $1401 \mathrm{keV}$ states of ${ }^{61} \mathrm{Fe}$

to other reaction mechanisms, such as the compound nucleus or the multi-step effect, which are evaluated using Hauser-Feschbach and coupled channel calculations, respectively.

\section{Knowledgments}

I would like to thank Prof Claudio Spitaleri and Dr. Gianluca Pizzone for their invitation to give a lecture in this wonderful summer school.

The two examples given in this contribution are $\mathrm{PhD}$ thesis works of N. Oulebsir and S. Giron. For the IPN-Orsay experiment, the work was performed in collaboration with my colleagues M.G. Pellegriti, P. Roussel, L. Audouin, D. Beaumel and S. Fortier from IPNO, A. Lefebvre, J. Kiener and V. Tatischeff from CSNSM and P. Descouvemont from ULB. For the Ganil experiment, the work was performed with N. de Séréville, P. Roussel and MUST2 collaboration people from IPNO, GANIL and CEA. So many thanks to my PhD students and to all my colleagues from IPNO, GANIL and CEA.

\section{References}

[1] T.A. Weaver and S.E. Woosley, Phys. Reports 227 (1993) 65-96

[2] M. Hashimoto, Prog. Theor. Phys. 94 (1996) 663

[3] F. Käppeler et al., Astro Journal 437 (1994) 396

[4] M.G. Pellegriti, F. Hammache, P. Roussel et al., Phys. Rev. C 77 (2008) 042801 and references therein 
[5] A. Belhout et al., Nucl. Phys. A793 (2007) 178 and references therein

[6] E. Kraussmann et al., Phys. Rev. C 53 (1996) 469

[7] F. D. Becchetti and J. Jänecke, Nucl. Phys. A 305 (1978) 293

[8] A. M. Mukhamedzhanov and R. E. Tribble, Phys. Rev. C 59 (1999) 3418

[9] Perey C. M. and Perey F. G. Atom. nucl. Data Tabl. 17 (1976)

[10] W. W. Daehnick et al., Physical Review C 21 (1980) 6

[11] G. R. Satchler. Direct nuclear reactions. Clarendon Press, international series of monographs on physics, 68 Ed., 1983.

[12] N. Austern. Direct nuclear reaction theories. Wiley-Interscience, monographs and texts in physics and astronomy Ed., 1970.

[13] I. J. Thomson et al., Comp. Phys. Rep. 7 (1988) 167

[14] J. D. Garrett et al., Nucl. Phys. A212 (1973) 600

[15] N. Oulebsir, F. Hammache et al., Phys. Rev. C 85 (2012) 035804

[16] N. Oulebsir, F. Hammache et al., AIP Conf.Proc. 1295 (2010) 254

[17] C. Matei et al., Phys. Rev. Lett. 97 (2006) 242503

[18] Smith et al., New Astr. ReV. 48 (2004) 87

[19] W. Wang et al., A and A 469 (2007) 1005

[20] K. Knie et al., Phys Rev Letter 93 (2004) 171103

[21] Mostefaoui et al., New Astr. Rev 48 (2004) 155

[22] M. Limongi and A. Chiefi, New Astronomy Reviews, Vol. 50, (2006) 474

[23] S. Woosley and Heger, Physics Reports, Vol. 442 (2007) 269

[24] S. Giron, F. Hammache, N. de Séréville et al., AIP Conf.Proc. 1213 (2010) 201

[25] R. L. Varner et al., Phys Rep 210 (1991) 57

[26] H. Krauss., Computer code TEDCA, (1995) 